macht bedingten Trennung der Bevölkerung in Berberophone und Arabophone; der von Frankreich befreite, selbständige Staat verdrängte die seinerzeit schnell französisierten Berber, schwieg ihre Existenz - wegen Kollaboration mit dem Feind - quasi tot und fühlte sich erst seit der Protestbewegung der kabylischen Berber im Frühjahr 1980 veranlaßt, diese Politik aufzugeben: Seit 1983 gibt es an der Universität Algier - wieder - einen Lehrstuhl für Berbersprache und -kultur. Inzwischen wird die Berberfrage in Marokko, Mali, Niger und Frankreich diskutiert.

Insgesamt weisen die Beiträge dieses Buches auf interessante Problemstellungen, bringen aber - bedingt durch ihre Konzipierung als Vorträge - nicht stets die notwendige Breite und Tiefe. Der zur Zeit des Kongresses noch unentschiedene Problemfall Iran und die sich hier im Zusammenhang mit der Schaffung einer Theokratie aufdrängenden Fragen sind - leider ausgespart.

Dagmar Hohberger

\title{
Eva Dietz
}

Der Funktionswandel der Koka in Bolivien

Spektrum (Berliner Reihe zu Gesellschaft, Wirtschaft und Politik in Entwicklungsländem) Nr. 26, Verlag Breitenbach Publishers, Saarbrücken, Fort Lauderdale 1990, 138 S., DM 21,--

Kokain ist die derzeit am weitesten verbreitete und am heftigsten diskutierte Droge. Nach ihrer raschen Verbreitung in den USA drängt sie derzeit mit Macht auf den europäischen Markt. Kokain und seine Bekämpfung gehören heute weltweit zu den vorrangigen Problemen.

Zusammen mit dem Kokain ist auch Bolivien, das neben Peru wichtigste Erzeugerland des Kokablatts (des Rohstoffs für die Kokainherstellung) in die Schlagzeilen geraten. Während die Wurzeln des traditionellen Kokakonsums im Andenraum vermutlich weit vor unsere Zeitrechnung zurückreichen, ist der Anbau des Kokablatts im großen Stil zur Rohstoffgewinnung für die Kokainproduktion kaum 30 Jahre alt. Die Beschreibung dieses Funktionswandels ist das Anliegen der vorliegenden soziologischen Diplomarbeit. Die Autorin kommt zu dem richtigen Ergebnis, daß dem traditionellen Gebrauch der Koka nach wie vor eine wichtige Funktion für die indianische Mehrheit der bolivianischen Bevölkerung zukommt: Kokablätter werden von den indianischen Bauem und Bergarbeitem vornehmlich als physische und psychische Hilfsmittel benutzt. Das Kauen der Koka hat eine hunger-, durst- und schmerzstillende Wirkung. Zugleich wirkt es als Aufputschmittel. Die Koka erleichtert somit das Leben unter extremen Klima-, Höhen- (und Arbeits-!) verhältnissen. 
Daneben - und unabhängig von der traditionellen Verwendung des Kokablatts - steht die Kultivierung des Kokastrauchs als kapitalistische Rohstoffquelle. Der Kokaanbau weitete sich aufgrund der explodierenden Nachfrage, aber auch aufgrund extremer Armut der bäuerlichen Bevölkenung in den vergangenen zwei Dekaden enorm aus. Als Folgen konstatiert die Autorin in erster Linie eine (weitere) Desintegration der bolivianischen Gesellschaft. Aufgrund verfehlter Wirtschaftspolitik blieb völlig verarmten Bauern und Bergarbeitem kaum eine andere Wahl, als vom Altiplano in die tropischen Täler und Ebenen zu ziehen und Koka anzubauen. Die zeitweise vom bolivianischen Staat geförderte oder doch zumindest geduldete Ausweitung der Kokawirtschaft verdrängte schließlich einen Großteil der legalen Wirtschaft - ein Aspekt, auf den die Autorin allerdings nicht weiter eingeht. Das Problem Kokain steht heute im Vordergrund der für das Land lebenswichtigen Beziehungen zu den USA und in zunehmendem Maße auch seiner Beziehungen zu Europa.

Die Autorin macht deutlich, daß es sich bei dem Kokaanbau zur Kokainherstellung in den andinen Regionen um etwas qualitativ Neues handelt. Damit kann die Arbeit einen Beitrag leisten, um die Diskussion über das Kokainproblem zu versachlichen und Vorurteile gegenüber Bolivien wie auch gegenüber anderen Andenstaaten abzubauen.

Heinrich Kreft

\section{Ingo Winkelmann}

\section{Kommunale Selbstverwaltung in Mexico}

- Das municipio libre: Wurzeln, Ausgestaltung und Wiederentdeckung einer in Vergessenheit geratenen Verfassungsinstitution -

Nomos Verlagsgesellschaft Baden-Baden, 1990, 415 S., DM 87,--

Diese aus der Freiburger Dissertation des Verfassers hervorgegangene Arbeit stellt in der Tat das erste umfassende deutschsprachige Werk zum mexikanischen Gemeinderecht dar. Ausgangspunkt ist die institutionelle Garantie des municipio libre in Art. 115 der Bundesverfassung von 1917 und dessen Reform 1983 in Erfüllung eines Wahlversprechens des Staatspräsidenten Miguel de la Madrid Hurtado. Der Verfasser arbeitet nicht nur die Verfassungsbestimmungen der Einzelstaaten, sondern sämtliche Gemeindegesetze auf, abgesichert durch Feldaufenthalte 1982 - 1984 in verschiedenen Gemeinden. Im Anschluß an Herbert Krüger (S. 33) offenbart sich auch hier in der politischen Wirklichkeit die wahre Staatsverfaßtheit. Mit selbst erhobenen Umfrageergebnissen wird das Selbstverständnis der in der Gemeinde tätigen Amtsträger und Bürger hervorgehoben.

Obwohl der Ruf nach dem municipio libre zu den Revolutionsgrundsätzen gehört hatte, hat sich dieses zum Verfassungsrechtssatz in Bund und Einzelstaaten erhobene Prinzip kaum mit einem prägnanten juristischen Inhalt gefüllt über das, was beinahe selbstverständlich 